\title{
Beyond Rhetoric to Poetics in IT Invention
}

\author{
Annie Gentes ${ }^{1}$ and Ted Selker ${ }^{2}$ \\ ${ }^{1}$ Telecom ParisTech, 46, rue Barrault, 75634 Paris, France \\ ${ }^{2}$ Carnegie Mellon University Silicon Valley, Mountainview CA \\ annie.gentes@telecom-paristech.fr, ted.selker@sv.cmu.edu
}

\begin{abstract}
Two kinds of discourse typically define scientific productions: logical (epistemology of science) and rhetorical (sociology of science). We suggest that research projects can also be analyzed as poetical productions. While rhetorical strategies anticipate controversies and deploy techniques to defend projects and findings, poetical practices deepen the cultural and symbolic dimensions of technologies. Based on use cases that show different ways the poetics come to bear on research and development projects in information technology (IT), we discuss the play on words and images and how they contribute to the definition and creation of a new technology within research projects. Three cases of poetical practices are presented: naming technologies, christening projects, and designing logos. We give examples of naming and project identity formation to underscore how such a poetic stance impacts projects. Images and words help people imagine what the technology is about by giving imaginary traits and cultural substance. This paper's analysis is a call for further work exposing the value of conscious use of poetical approaches to deepen the framing of IT projects.
\end{abstract}

Keywords: Rhetoric, Poetics, Project Definition, Design, Sociology of Science, Sociology of Technology, Invention.

\section{Introduction: From the Rhetoric to the Poetics of Science}

Science endeavors to create and test hypotheses [1]. Scientific contribution is then based on logical demonstrations that distinguish it from social and other common knowledge discourses. The epistemologies of sciences detail the methods of validation of what they build as facts, either true or false. Inductive and deductive methods are discussed. But science is also about strategies to defend a territory, to plead for a cause, to affront enemies, to gather followers. This strategic part of science has been extensively analyzed by the sociology of science after Latour [2]. Actors build a full-fledged rhetorical strategy to support their project. The sociology of sciences and technologies [3] has shown that making science implies defending it and therefore elaborating a rhetoric that is going to convince the stakeholders. The father of rhetoric, the Greek philosopher Aristotle, describes rhetoric as an art of persuasion that every citizen should learn so as to debate about things in the city. In society, things are being discussed and judged. Winning the audience is the focus point of rhetoric. Latour's contribution has been to analyze the form and role of scientific rhetoric. 
In particular, he shows that scientific papers are built around a defensive strategy that finds allies as a way to prevent dismissal. He describes how researchers use rhetorical arguments from theoretical background, "enrolling" other papers that have been written so as to prevent attacks. Latour speaks of "stratification" [2] that is to say the building of a system of authority within the paper. This system also excludes those researches that do not follow the same direction. Research and scientific activities are therefore described like a court of law, where participants build up the defense of their case. For Latour, this rhetorical strategy makes it difficult for scientists to produce certain types of discourses. In particular, he points out that popularization is difficult because scientific texts are written to exclude people not to include them. What is more, scientific controversies need strong positions that do not leave space for dreams or imagination.

However, certain texts (including images like drawings, photographs and videos) that are produced in the course of a research project cannot be totally described by either their deductive or inductive building of facts, nor their rhetorical function. Why call a technology Bluetooth after a king of Denmark? Why choose a cartoon character - Popeye - to represent an adhoc mobile communication research project? And why tell all kinds of stories when one might expect to communicate technical information by concentrating on algorithms and material implementations? We suggest the following answer: research done to study an area and engineering done to create technologies are not only based on logical demonstrations, or by negotiation between actors, they benefit by being subjective and cultural associations, poetic activities related to humanistic and literary practices too. They are using and working on symbolic representations that expand the observations and definitions that researchers obtain within their methodological framework and constitute an alternative to the rhetorical inscription in society by a poetical invention of concepts and narratives. Though we describe practices that have been experienced by most researchers in HCI and engineering, these practices can be analyzed as an integral part of the research process rather than discarded as communication, or valorization practices. Our standpoint is that these practices that focus on the subjective and cultural associations need to be considered as an essential part of the technical research as an invention. We consider invention along Simondon's definition. The philosopher of science and technology defines invention by articulating imagination to cultural and social context through a tangible artifact [4].

This article contrasts the poetical and the rhetorical, and their role in the emergence of new technologies. We contend that engineers, designers and scientists expand their work with their literary and humanistic skills that we propose to call their poetic stance. Ignoring the poetic aspects of their work is detrimental to a clear understanding of how it comes about. It could also be detrimental to strategies of innovation that have to take into consideration the whole gamut of activities around invention and creation to make sure that the potential of technologies are explored.

Our goal here is to show the value of poetics in IT projects. We first start from the poetic stance from Aristotle's founding text and a recent body of literature on poetics. We then present several use cases that are samples of this poetic stance: naming of technology, christening of a project, and design of logo. The analysis of these use cases shows interactions between the poetic naming and narrative process (with words or images) and the projects themselves. 


\section{A Poetic Stance}

Before analyzing some of these poetic productions of science, we propose to start on the basis of Aristotle's definition of "poetics" and how it has been developed in literary theory in order to understand what is at stake in these images and stories that use more fictional metaphors and concept visualizations than either fact based demonstrations or rhetorical arguments [5]. Then we proceed to support our argument for poetic practices with examples of these poetic productions.

Like rhetoric, poetics is an art of production. While over the centuries, poetics has been reduced to a collection of stylistic traits and can still be considered as such (the poetics of literary genres, like science fiction for instance) more recently, poetic studies have focused on the creative process itself and its specificity [6]. In particular, the linguist Jakobson proposes a model of language that takes into consideration its different functions. Among them, the poetic function of language refers to itself, in other words is its own material. It is opposed to the referential function of language that points to things [7]. Referential and poetic functions of language are further developed by semioticians, who distinguish denotation and connotation. Denotation is about the literal meaning and essentially descriptive while connotation is the subjective and cultural associations [8]. The poet and literary analyst Paul Valéry described this poetic stance happening when the purpose is not to use language as a persuasive means to create a social consensus but to transcend the here and then, because the writer or artist unfolds either latent or possible aspects of the world [9]. For Meschonnic too, specialist of poetry and translation, the creator questions what could be [10]. This production can be called prospective, not because it foretells the future but because it produces new meaning. Aristotle uses the word Dunamis or dynamis that means possibility or capability. It is not an abstract vision. The design work consists of processing, working on, and with a variety of materials: either language (styles, genres [11], or pigment or sound [12]. Playing with words, inventing stories, making images are not only there to teach, or to defend, or to demonstrate. They expand the way we think about the world. The artist taps into and reveals the potential of a medium to transform and interpret the world.

Such inventive practices within research projects have recently been the subject of social studies of scientific texts [13], in ethnography for instance [14], and of imaging and visualization. Scientific images, for instance, have been noted for their aesthetic ambition [15]. Sociologists of science, Burri and Dumit point out the "engagement" that images produce and in particular how they are made instrumental in the production of scientific knowledge [16] While Latour [2], Lynch and Woolgar [17] stress how images tend to objectify knowledge and to produce yet another argument in the rhetoric of science, we follow those who note the fecundity of images with respect to creativity and invention. Images in this respect are considered as epistemic creations [18].

While the theme has been thoroughly studied in different fields of science, we think that it needs further analysis for at least two reasons. First we need to identify the diversity of poetic productions - not only images of science, but a whole gamut of other productions like names and logos. Second, we need to question their relationships and their impact on the project. 


\section{$3 \quad$ Case Studies}

\subsection{Methodology}

To study the poetic stance of inventors, designers and engineers we combined two methodological traditions. The authors participated and observed research projects from their inception to their completion and could therefore study the way names and narratives played a role in the maturation of the invention. These observations were eventually completed by in-depth interviews. These methods from social sciences, such as ethnographic observations and participations as well as interviews were complemented by methods from the humanities: literary analysis of texts, semiotic study of documents, analysis of media. These analyses focus on meaningmaking as defined in semiotics [19], anthropology of material culture [20] and more recently design studies [21]. They study the references to certain literary or visual traditions that are used within videos, texts, photos. These analyses show how words and tales not only can describe the technology, but also carry the imagination of the technology rather than its facts, and in doing so influence the way researchers look at their projects.

Several aspects of this poetic process are exposed in this article: the question of naming the technologies in the view that they will become common names; the christening of projects with proper names, and the role of logos.

We defined a corpus of texts related to research projects that either author was taking part in. Semiotics scholars define a corpus as a group of texts (linguistic and visual) that belong to the same genre [17]. Texts of the same genre share structural and pragmatic characteristics in so far as they are not only similar in style, narrative structure, or visual organization, but also as they are discussed by social actors as targeting the same audiences, for the same purposes, with similar textual strategies. The constitution of the corpus does not pretend to be exhaustive. It is always but a segment of the actual production. The purpose is to gather enough material to answer a research question. The method therefore consists in defining a corpus of textual elements and considering what are the shared elements and their purpose in the text. In this particular instance, the authors selected only part of the poetic elements of the research practice". They focused the semiotic analysis on condensed forms of identity of the technology, of the group, through naming or representing by a logo over a diversity of situations: naming a field of research, a device, a communication norm. The purpose is to emphasize the similarities over different examples rather than to focus on the differences that should be further explored.

\subsection{Scientific Puns: Play on Words and Definitions}

In 1982, the three volumes AI Handbook attempted to present all of published AI projects of the time [22]. At that time the corpus consisted of 50 or so named research 
systems ${ }^{1}$. They were named with words or acronyms that punctuate the index with a variety of poetic heritages. WHY is a question answering system, BUGGY is a system to help students debug their understanding; some 30 such as WHY, BUGGY or Scholar use functional and denotative names. 20 more of the research project names such as CRYSTALIS or MYCIN are clearly connotative whimsical names words to make them memorable or celebrate some joke that have no bearing on the function of the programs. 8 of the names such as SOPHIE could be easily given a person. As this simple retrospective of the AI handbook shows, first there is a variety of naming options, and second many of them are visibly not pushed by purely descriptive purposes.

More generally, some words like 801.11 describe the way the technology came into being. Some words like "wi-fi" for supposedly "wireless fidelity" are the result of brand work. Others like " mesh networks" rely on metaphors that seems to relate to a physical network. Still other names completely drop out from the technical description to refer to an apparently arbitrary choice as the term "Bluetooth". In, this article, the central methodology follows the ethnographic approach of identifying concepts from an analysis of specific example projects. Here we study three examples that seem to belong to different naming strategies: "Bluetooth" as a private joke within a community, "Artificial Intelligence" as relating a technology to artificial beings in literature, "TrackPoint" as renaming in a lineage of products.

Bluetooth. Bluetooth as a name could have come from many places around the time of its creation. Blue lasers and LED's were the high-tech discoveries that were being used to sterilize things, to drive higher bandwidth on laser disks and optical fibers. But none of these references had the least to do with the short range Bluetooth radio standard. The term "Bluetooth" came out of meetings between researchers who were attending normalization meetings on the issue of short-range radio frequency. A leading actor of the "Bluetooth" norm, Jim Kardach, posted on his blog that the diversity of technical names was such that it led to use a code name from the culture of some of the participants [23]. The name King Harold Bluetooth of Denmark, who succeeded at unifying the various Danish regions in the 10th century, had emerged in discussions and post conferences.

1 The 48 prominent AI research projects of the time are described in the1983 AI handbook [22]. The names of these research projects can be classified as, A: a person's name, B: connotative/whimsical or arbitrary, or C: functional.

A. 8 of the 48 names could easily be used as a person's name without notice: DEDALUS, GUIDON, HAM, NOAH, PECOS, SOPHIE, TEIRESAIS, WUMPUS. B. 20 of the names are connotative whimsical or arbitrary:

ACRONYM, CRYSTALIS, CASNET, DEDALUS, GUIDON, HAM, HARPY, IRIS, LIBRA, MYCIN, MEMOD, MOLGEN, NOAH, PECOS, SAFE, SOPHIE, STRIPS TEIRESAIS, WEST, WUMPUS.

C. 28 of the projects are named with functional or denotative names:

ABSTRIPS, ACT, Alpha beta, AM, AQ11, BUGGY, CHI, CONGEN, DENDRAL, EPAM, EXCHECK, EXPERT, HACKER, HEARSAY, HWIM, INTERLISP, LEX, Meta-DENDRAL, MACYMA, Minimax, NLPQ, PROSPECTOR, Protosystem, PSI, SCHOLAR, Student, The Programmers Apprentice, WHY. 
"Bluetooth was borrowed from the 10th century, second King of Denmark, King Harald Bluetooth (King Harald I of Denmark) who was famous for uniting Scandinavia just as we (Intel, Ericsson, and Nokia) intended to unite the PC and cellular industries with a short-range wireless link.” [21]

Rather than keeping complicated names or acronyms that were far too descriptive and technical the participants chose the name Bluetooth that started as a joke and finished as the expression for the norm....

"...it became apparent the need to have a single name; as Intel would talk to people about "Biz-RF", Ericsson about "MC-Link", and Nokia about "Low Power-RF", which also created confusion..." [21]

While it was not meant to be the final name, it ultimately ended up becoming the official name of the standard.

TrackPoint. Another example is the "TrackPoint" device. According to its inventor, Ted Selker, it was the subject of multiple debates. At IBM, several years were spent developing a way of moving a cursor without moving hands from the home keyboard position. The project was first called the "Jstick" for the use of the right index finger on the $\mathrm{j}$ key as a joystick. Some people picked up this solution, included it in a few laptop computers in the mid 1990's, and marketed it as "Jmouse".

Due to problems with discoverability, installation, button overloading and handedness, the device moved out from under a key to between the $g$ and $h$, and got christened with a functional name: "pointing stick" in press release and presented at Interact1990 [24]. Various people proposed that if a 6 inch handle that users wiggled was named a joystick this little one could be named "joy nub”. As IBM's design consultant Richard Sapper chose red for the device, many thought of calling it a clitoris or a nipple. Scientific naming conversations focused on an easy way to remember the descriptive "objective" name, but product naming conversations focused on a descriptive "subjective" name. In the objective case, its origin in some early experiments under the $\mathrm{j}$ had people calling it the "Jstick", the underlying technical system being an isometric joystick and its use as a moving a cursor or focusing on a point on the screen got it called a "pointing stick". In the subjective case, people described the appearance and the gesture and considered it as obviously erotic. As the device was about to go to product, the naming department of IBM gave it a different name which turned out to have a trademark problem after the launch team was already being trained. There were tense conversations with the naming person who thought naming this little feature was trivial as he had whole product lines and products to name... With only weeks till public communications were to begin, he chose a recycled name: TrackPointII. The name TrackPoint had been trademarked as a descriptive name for a mouse product that IBM made in 1991 [25] that could be turned over to be used as a trackball that a user rolled their hand over to move the cursor. The term track also changed the connotation: from a person's actions of rolling a ball to the person's goal of tracking down a point or pointing at the screen. In both devices, the "point" concentrates on the effect on the screen. 
Artificial Intelligence. Finally, in interviews with Selker, the late Professor John McCarthy explained how he picked up the name for a new field: "Artificial Intelligence" in the 1950s [26]. McCarthy was a huge fan of science fiction throughout his life. In many conversations he would state that he had thought Artificial Intelligence (AI) would captivate people's imagination more than the other term he had been considering: "Machine Intelligence". Embedded in his choice of name was his love of science fiction but also the fact that he supposed that people would be more interested in exploring human intelligence as well as more capable machines and he wanted to connect the two. Instead of simply describing the work as making yet another engineering field, he liked the Artificial Intelligence expression for its poke at anthropomorphizing the quest.

\subsection{Naming Projects: Acrobatic Acronyms}

Technologies that are invented need to be given a name. Similarly, though for a shorter period of time, research projects are also baptized. There too, one can witness a variety of inspirations: eventually project participants may forget what the name of the project stands for. One of the reasons is that they are made of acrobatic acronyms. In fact, people often keep the acronym but change its explanation. Some names are more telling than the others: PLUG ${ }^{2}$ (Play Ubiquitous Games and Play More) that was chosen for an RFID mobile game sounded both relevant and evocative. "Transhumance $^{3 ",}$ "Safari" ${ }^{4}$ " that focused on adhoc networks, were suggestive of mobility, but did not give any real clue to how they were dealing with it. And of course, "Adam"

or "Popeye", are particularly obscure.Gentes could observe the naming process for a research project carried out by two departments of social sciences (Center for the Sociology of Innovation in Ecole des Mines and the Co-design Lab at Ecole des Telecom) as well as a legal research lab (CERNA). The team wanted to study the uses of peer-to-peer but also mobility and ad hoc networks. They wanted to understand how these technologies were spreading in our society. In fact, a good part of the discussion was about the object of research itself. For a part of the team, peer-to-peer was a good starting point, but it focused on applications, while the other members wanted to focus on infrastructures. Finally, the common denominator between the two groups was the fact that they were interested in Distributed Architectures. That sealed at least 2 letters of the acronym that the team was looking for: AD. But Distributed Architectures is a much broader field than a project around communication could own. Starting with $\mathrm{AD}$, they played on a number of combinations based on the sonority and the connotations of the words: AD + mobiles information: ADMIN that gave the project the connotation of computer management. The team tried: AD+ Evolution_of Relations:

${ }^{2}$ PLUG - play ubiquitous games- ANR/RIAM -2007-2009.

3 Transhumance (Ad Hoc Services: Fulfilling a Platform for Peer to Peer Applications on Mobile Ad Hoc).

${ }^{4}$ Safari - Mobile Ad Hoc Networks - ANR/RNRT- 2003-2005.

5 Architectures Distribuées Pour Applications Multiples,

http: / / adam. hypotheses.org/

${ }^{6}$ POPEYE (Professional Peer Environment Beyond Edge Computing) - STREP/IST - 20062008. 
ADER. But the group promptly remarked that it sounded like the inventor Clément Ader who was a major inventor in the aviation field and also for the telephone. AD + Ingénierie des Relations_(engineering of relations): ADIR. But none of these names were "fun" and the group finally came up with ADAM that would stand for "Architectures Distribuées Pour Applications Multiples" (distributed architecture for multiple applications), that did not mean much more than the other options but that combined applications with infrastructure and a biblical name, knowing that at the time there was only one man in the team.

\subsection{Scientific Logos: A Question of Identity}

The logo is a sign of branding permeating the logic of research as a commodification process [27] that "mediate the junctions between technological producers and consumers". Our view is that this trend of naming is not only functional, memorable and strategically positioning the project but also poetically adding to the identity of the project.

We would like to go back to one particularly successful example that was, in effect, realized by a professional designer, while most of the time new project teams and research groups try to find the resources within their team, students, or friends who agree to do it for free...

The project is called ILHAIRE: Introducing Laughter in Human Avatar Interaction, Research and Experiment [26]. The logo was realized by one of the research partners, the French animation company: La Cantoche. Though it is not a communication company and it does not do logos for a living, they have graphic designers and artists who could work on the project. The whole process is interesting in as much as it is quite different from an ordinary process of branding. According to the project manager, one of the main differences is that the team includes "a large variety of disciplines and interests in the project and there is no formal hierarchy between the members [interview with J.]" so making a decision was perhaps more complicated in that respect. Another difference is that the logo is defined in relation to the theme of the project (and not as could be the case in other circumstances to position the "product" within a market). What makes it really precious then is that, according to J., "it focuses on the identity of the project, the "heart" of the project".

The exchanges between the different participants showed that this identity was a subject of controversies: social scientists contended that laughter was social, for others it was important to see that laughter was necessary to health and well being. Engineers focused on the models of gestures and acoustic dimensions. The team was also worried that talking about laughter would not be taken seriously. They wanted to make it clear that it was research, which was finally why the baseline was chosen: "the science of laughter". The production of the logo was therefore one of the productive and possibly rare occasions where the different stakeholders explained and compared their viewpoints.

What makes it a real poetical work is that the participants had to learn about the process itself, the impact of images, the role of signs and words. The role of the project manager was particularly important in sharing this vision about the making of 
a logo. For instance, they had to understand that a logo is "not an illustration". In particular, it was tempting to use an avatar as part of the logo but then it would work as an example, rather than a sign with a more general purpose. They also had to work on how to share values through the choice of a logo: "warm colors", "playful", or showing: "the collaboration between different fields of research (engineering + human sciences) [28]".

The process went from a culture of words with denotative value to a process of images with an emphasis on culture- from trying to deploy the acronym, to gradually incorporating the culture of cartoon, with references to the field of computer science.

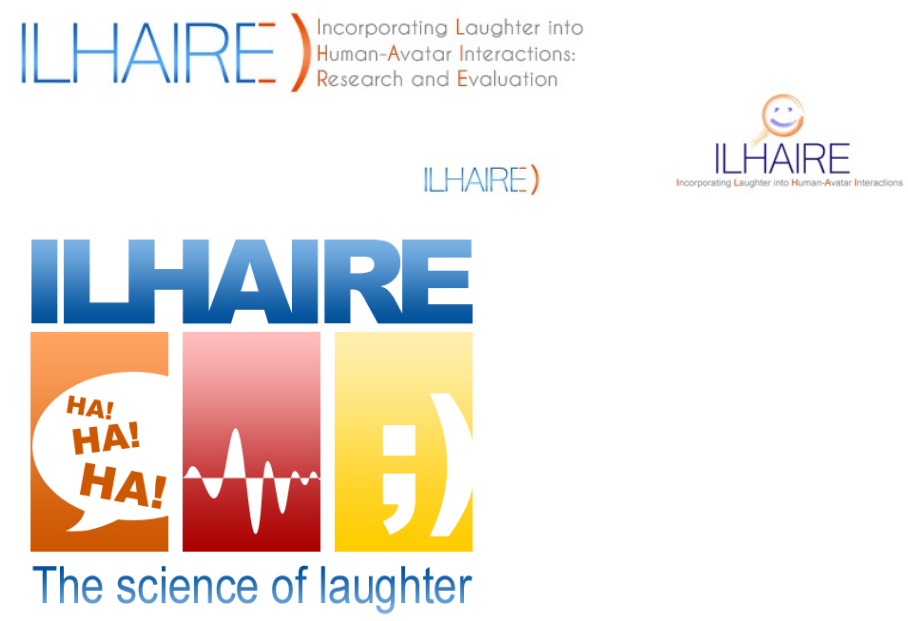

The final logo shows a mastery of graphical techniques: see for example, the bubble and emoticon that are left open by the frame of the rectangles.

But more importantly, it played a role in the representation of the project for the participants. It crystalized a partnership, an identity, and the representation of a technology.

\section{$4 \quad$ Findings}

Despite the differences between giving names to technologies, to projects or designing a logo, the examples in this paper emphasize the relationship between a technology and the different ways to invent it.

Names are caught between a denotative and connotative tension. In the naming process, the example of the TrackPoint naming story as told above is an epic journey of descriptive functional names (pointing stick) and descriptive joking names with sexual innuendos. Despite the differences, the top three options ("pointing stick", "joy nub", "TrackPoint") struggled with an accurate description of the device. The name either points to the underlying technology or to its surface properties, how it works 
or how it is perceived from a user perspective. The difficulty to choose between denotative options: pointing stick and TrackPoint, shows that to describe something might not be so easy in the first place. As demonstrated by Bucciareli [29], designing is about agreeing on a definition; different stakeholders have a different definition of what the technology is about. What this example as well as the Bluetooth example show is that the denotative choice is not solving the problem and that names are produced also with connotations in mind. This is obvious in both "Artificial Intelligence" and "Bluetooth" as well as the discarded "joy nub". Connotations deepen the cultural relationship of the technology with other artificial products because they evoke linguistic and social codes, rhetorical and ideological conventions and therefore enlarge the cultural references of the innovation. Play on words are used to explore and exploit connotations and support associations of ideas in the case of naming technologies or projects. As related by its inventor, "joy nub" was probably the more striking and memorable name, but was abandoned for reasons of propriety. Artificial Intelligence was picked by its author with a background of at least two centuries of literature on artificial creatures. What was at stake was to stimulate the imagination and the research thanks to the evocative metaphor of intelligence and the open ended nature of the word artificial that no longer related the expression to an actual artifact but acted as far more diffused.

Names are also compared to other names and finding the right name might have to do with the necessity to break from or on the contrary to establish a connection with other names. Trackpoint (at the beginning called Trackpoint II) was chosen to establish such a connection. It was thought of in relation to other existing products or devices. The naming process therefore focused on founding the new device within a family, and gave it a sense of continuity, an inscription in the history of tracking devices. Part of the imaginative process was about the lineage of technical artifacts. On the other hand, Bluetooth does not relate easily to any other existing technology and is strikingly original as it decidedly departs from any denotative and descriptive naming tradition.

Giving a name to a project seems to be slightly different from giving a name to a technology. While the inventors of a technology aim at creating a common name, the groups who baptize their project inscribe their activity in history and identity through a proper name. Names given to project can be chosen to be descriptive, to remind people of the roots of or aspirations for the project, or simply be a memorable word or sound that will attach to people minds to help the project. According to Kripke [30] this proper name stands for the dubbing ceremony that accompanies its creation. As proper names, they have some relationships with the culture of their authors: Bluetooth is chosen by people mostly coming from Nordic countries, Adam is a private joke about the only man of the research team. The connotations are then exploited to signify not so much the technology as the collaborative work of the team. But the word then does not so much refer to anything (who really knows who Bluetooth was and what he did?) than build on the originality of the choice of words that appear truly poetic if not totally random: a tooth: something tiny but piercing, associated with the unlikely colour blue that might refer to a range of other technologies of the time (Blue Ray for instance). While proper names are the subject of debates between descriptive 
or causal historical theories [31], an important point is that they are also contextsensitive [32]. This means that they can take on meanings very differently according to the context of utterance. In fact, a proper name invites different viewpoints on the same thing because it is not purely descriptive: for each partner of the ADAM project, the identity of the project remains open to interpretation. In fact, the combination of Distributed Architecture on the one hand, and Multiple Applications on the other while originally chosen for evocation purposes, also forced the members of the team to think about the relationship between infrastructures, applications and services. It expanded the conceptualization of these systems. As a proper name, they benefit from a certain "looseness", as defined by Searle [33]. They do not describe and give attributes to an object (or person) but still refer to it in ways that open to a whole family of possible descriptions. A literary analysis of these names shows that they try to mix technical features with a symbolic representation of the technology.

Designing logos apparently shares some of these issues of group identities and technological descriptions as the example of Ilhaire tends to show. The image is chosen to represent the technology without using the usual tools of the trade, like scientific schemas. Rather the example shows that several cultural relationships are designed within the iconic choices. The logo is clearly related to the culture of cartoons. It also alludes to emoticons and finally to scientific images through the representation of a sound curve. It functions as a metonymy: the project is part of a wider scientific, cultural and literary whole. Similarly to the expression "Artificial Intelligence", it therefore opens up to larger realm of possible research and applications.

\section{Conclusion}

In this article, examples bring forward the value of imagination and strive to promote the study of poetic practices in research project. These examples show that we can use methods that combine sociological methods and methods from humanities to produce thorough analyses and comparisons and come up with typologies of poetic practices integral to all IT project. The primary function of these writing practices is not only descriptive. Names of technologies, acronyms for projects, logos and narratives, are motivating, aspirational and evocative practices that support the expansion of the scope of the technology.

Poetics can be understood in production and in reception. In production, the poetic work is an aesthetic and intellectual work that looks for meaning. These productions, rather than working on precise attributes, operate what the logician and philosopher of language Saul Kripke, calls the "opening of possible worlds". Even in the case of technology naming that tends to limit the possible connotations, we have seen that the exploration of names becomes an expansive part of a project with results hovering between denotation of what the project is about and connotations of how people want to feel about it. In reception, the proper name of a project is also poetic, because like other proper names, it supports a context-sensitive diversity of interpretations. The Artificial Intelligence example above shows how the diversity of interpretations might be designed into a name to help the project to continually expand its goals. 
Poetics as part of the aesthetic experience depends on the process of open interpretation. A word or an image that seems to close the object on its properties does not support the same interpretive leeway. We can use the difference that Umberto Eco [18] analyzes between a traffic sign and a work of art. The traffic sign is no longer a traffic sign if it is freely interpreted and discussed. But a work of art constitutes "an open product on account of its susceptibility to countless different interpretations which do not impinge on its unadulterable specificity. Every reception of a work of art is both an interpretation and a performance of it, because in every reception the work takes on a fresh perspective for itself [18]. Therefore, what is at stake in these poetical practices of engineering is to explore the cultural meanings of the technology, then to open it to a diversity of interpretations.

This paper focuses on the semiotics of condensed forms of identity through name and logo. But poetic practices include other literary and visual forms that can affect research projects. In particular, we can concentrate on narratives such as use-cases or personas and the way they contribute to build and expand the scope of research projects. We could also compare these findings to research projects over time. Placing this research within a historical perspective would help us to consider different poetic practices, different genres and styles over time. For instance they would help to figure if the growing multidisciplinarity in research projects is a key factor in the production of figures of speech that gather the different disciplines. In further research, we can also explore how words and images that accompany the emergence of a technology not only help researchers anchor their technology in culture, but also contribute in special ways to the involvement of potential users. In particular, when users are asked to test technologies, how do they interpret them based on a diversity of factors including their names and other representations. We should further enquire into how these representations help structure a design for debate around its value and use [34]. This research program can help to establish a taxonomy of poetic practices according to fields of research, actors, types of research products. Beyond the taxonomy, a theory of a poetic of research can help the process of invention by articulating findings to an artifact situated in and incorporating social and cultural context.

\section{References}

1. Chalmers, A.F.: What Is This Thing Called Science?, 3rd edn. Open University Press (1999)

2. Latour, B.: Science in Action: How to Follow Scientists and Engineers Through Society. Harvard University Press (1988)

3. Hackett, E., Amsterdamska, O., Lynch, M. (eds.): The Handbook of Science and Technology Studies, 3rd edn. MIT Press (2007)

4. Simondon, G.: L'invention dans les techniques. In: Cours et Conferences. Seuil (2005)

5. Sachs, J. (trans).: Aristotle: Poetics. Focus Publishing/ R. Pullins Co. (2005)

6. Preminger, A., Brogan, T.V.F., Warnke, F.J. (eds.): The New Princeton Encyclopedia of Poetry and Poetics, 3rd edn. Princeton University Press (1993)

7. Jakobson, R.: Linguistics and Poetics. In: Sebeok, T. (ed.) Style in Language, pp. 350-377. M.I.T. Press (1960) 
8. Barthes, R.: Elements of Semiology. Lavers, A., Smith, C. (trans.). Jonathan Cape, London (1967)

9. Valéry, P., Oeuvres, G.H.J. (eds.): Collection Pléiade, Variétés, Paris, vol. 1 (1957)

10. Meschonnic, H.: Pour la poétique, Gallimard - Nouvelle Revue Francaise, vol. 1 (1970)

11. Genette, G.: Introduction à l'architexte. Seuil, Paris (1979)

12. Gentès, A.: The Design of Emotions: How the Digital is Making us More Emotional. In: Pelachaud, C. (ed.) Emotion-Oriented Systems, Hermès, London (2011)

13. Olohan, M.: Scientific and Technical Translation. In: Millan-Varela, C., Bartrina, F. (eds.) Routledge Handbook of Translation Studies, Routledge, London, New York (2012)

14. Clifford, J., Marcus, G.E. (eds.): Writing Culture: The Poetics and Politics of Ethnography. University of California Press (1986)

15. Sicard, M.: Chercheurs ou Artistes? Entre Art et Science, Ils Rêvent Le Monde, Autrement (1995)

16. Burri, R.V., Dumit, J.: Social Studies of Scientific Imaging and Visualization. In: Hackett, E., Amsterdamska, O., Lynch, M. (eds.) The Handbook of Science and Technology Studies, 3rd edn., pp. 297-317. MIT Press (2007)

17. Lynch, M.E., Woolgar, S. (eds.): Representation in Scientific Practice, 1st edn. MIT Press (1990)

18. Cambrosio, A., Jacobi, D., Keating, P.: Ehrlich's 'Beautiful Pictures' and the Controversial Beginnings of Immunological Imagery. Isis 84(4), 662-699 (1993)

19. Eco, U.: The Open Work. Harvard University Press (1989)

20. Keane, W.: Semiotics and the Social Analysis of Material Things. Language \& Communication 23, 409-425 (2003)

21. Krippendorff, K.: The Semantic Turn, A New Foundation for Design. Taylor \& Francis, Boca Raton (2006)

22. Barr, A., Feigenbaum, E.: The Handbook of Artificial Intelligence, vol. 1. William Kaufmann, Inc. (1981)

23. http://www. eetimes.com/electronics-blogs/other/ $4394006 /$ Bluetooth-how-it-almost-didn-t-happen-

24. Rutledge, J.D., Selker, T.: Force-to-Motion Function for Pointing. In: Proceedings of INTERACT: IFIP International Conference on Human-Computer Interaction, Amsterdam, Netherlands, pp. 701-706 (1990)

25. Trackpoint (Model L40 SX) Manual, Publication no. 1397090 (1991)

26. McCarthy, J., Minsky, M.L., Rochester, N., Shannon, C.E.: A Proposal for the Dartmouth Summer Research Project on Artificial Intelligence, http: / /www-formal. stanford.edu/jmc/history/dartmouth/dartmouth.html

27. Fishman, J.R.: Manufacturing Desire: The Commodification of Female Sexual Dysfunction. Social Studies of Science 34(2), 187-218 (2004)

28. http: //www.ilhaire.eu/

29. Bucciarelli, L.L.: Designing Engineers. MIT Press (1996)

30. Kripke, S.A.: Naming and Necessity, New edn. Wiley-Blackwell (1991)

31. Brock, S.: The Ubiquitous Problem of Empty Name. The Journal of Philosophy 101(6), 277-298 (2004)

32. Burge, T.: Reference and Proper Name. The Journal of Philosophy 70(14), 425-439 (1973)

33. Searle, J.R.: Proper Names. Mind (67), 166-173 (1958)

34. Gaver, B., Dunne, T., Pacenti, E.: Design: Cultural Probes. Interactions 6(1), 21-29 (1999) 